\title{
An analysis of the incidence of adverse pregnancy outcomes among women with psoriasis in Newfoundland and Labrador
}

\author{
Dr. Wayne Gulliver ${ }^{1,2}$, Andrea Morrissey ${ }^{3}$, Shane Randell ${ }^{1,2 *}$, Susanne Gulliver ${ }^{1}$, Dr. Don MacDonald ${ }^{2,3}$ and Catherine Wright $^{3}$ \\ ${ }^{1}$ Newlab Clinical Research, St. John's, NL, Canada \\ ${ }^{2}$ Department of Medicine, Faculty of Medicine, Memorial University of Newfoundland, St. John's, NL, Canada \\ ${ }^{3}$ Newfoundland and Labrador Centre for Health Information, St. John's, NL, Canada
}

\section{Introduction}

A retrospective cohort analysis was conducted to compare the incidence of adverse birth outcomes (Caesarean delivery, small for gestational age, low birth weight, preterm birth, and congenital anomaly) among women with and without a diagnosis of psoriasis who gave birth in Newfoundland and Labrador between 1992 and 2012. Linked psoriasis patient data and birth data were used to assess the associations between psoriasis case/control status and disease severity (mild or moderate-to-severe) and pregnancy outcomes.

\section{Background}

Psoriasis is a chronic, debilitating inflammatory disease characterized by lesions of the skin [1]. Worldwide, estimates for the prevalence of psoriasis in adults range from $0.91 \%$ to $8.5 \%$, with higher prevalence estimates reported at higher latitudes [2].

Onset of psoriasis has a bimodal age distribution: the earlier peak, which is associated with more severe disease, occurs between ages 15 and 30, and the later peak between ages 50 and 60 [3]. The disease therefore affects many women of childbearing age [4-6]. In the United States, an estimated 65,000 to 107,000 births to women with psoriasis occur each year, approximately 9,000 to 15,000 of which are to women with moderate-to-severe disease [7].

The inflammatory processes underlying the development of psoriasis may adversely affect pregnancy outcomes. Many of the histopathological changes observed in the skin of psoriasis patients are thought to be due to the action of proinflammatory cytokines [8]. Elevated levels of TNF- $\alpha$, interferon- $\gamma$, and IL- 6 have been detected in serum and skin of psoriasis patients, and are correlated with disease severity [9]. It has been postulated that these cytokines may adversely affect the placenta, thereby leading to impaired fetal growth and low birth weight $[10,11]$.

Further evidence in support of an increased risk of adverse pregnancy outcomes resulting from the inflammatory processes that characterize psoriasis is provided by studies of other immune-mediated diseases. Higher rates of low birth weight, small for gestational age, Caesarean delivery and preterm birth have been observed in women with inflammatory bowel disease [12-14] and rheumatoid arthritis [1517].

Nonetheless, little is known about the effect of psoriasis case status and severity on pregnancy outcomes. Prior studies have examined psoriasis in relation to numerous pregnancy outcomes, including preterm birth $[6,18]$; low birth weight $[6,18]$; size for gestational age $[5,18]$; Caesarean delivery $[6,18,19]$; hypertensive diseases of pregnancy, including pre-eclampsia or eclampsia $[5,6,18,19]$; premature rupture of membranes [5]; macrosomia [5]; and miscarriage or recurrent miscarriage $[5,6,19]$; but their findings are contradictory, and several have been limited by small sample sizes and potential exposure misclassification [6]. Further, few studies have explored the effect of disease severity on outcomes [5].

In the current study, we sought to examine the effect of psoriasis case/control status and severity on several outcomes of pregnancy, including method of delivery, gestational age, size for gestational age, birth weight, and presence of congenital anomaly at birth.

\section{Research objectives}

The objectives of the study were as follows:

1. To describe the demographic (marital status, urban/rural residence, education) and reproductive (parity and age at first birth) characteristics of women with and without psoriasis.

2. To examine overall rates of adverse pregnancy outcomes among women with and without psoriasis.

3. To examine overall rates of adverse pregnancy outcomes by psoriasis severity (mild or moderate-to-severe psoriasis versus controls).

4. To analyze psoriasis as a predictor of pregnancy outcomes, controlling for potential confounding factors.

5. To analyze psoriasis severity as a predictor of pregnancy outcomes, controlling for potential confounding factors.

\section{Methodology}

\section{Study design}

A retrospective cohort study design was used to analyze pregnancy outcomes among women with and without psoriasis, by case status and

Correspondence to: Shane Randell, Newlab Clinical Research, St. John's, NL, Canada, Tel: 709-752-6000; E-mail: srandell@newlabresearch.com

Received: May 20, 2015; Accepted: August 13, 2015; Published: August 17, 2015 
disease severity. Medical records of psoriasis patients at a dermatology clinic (NewLab Clinical Research Inc., St. John's) were linked via a unique identifier to a dataset of live birth registration records in Newfoundland and Labrador, and the associations between psoriasis case status and disease severity and the incidence of Caesarean delivery, Small for Gestational Age (SGA) birth, Low Birth Weight (LBW), Preterm Birth (PTB), congenital anomaly, and overall negative pregnancy outcome were analyzed.

\section{Data sources}

The NewLab Psoriasis Clinical Database is a database comprising medical, demographic, and genetic data on psoriasis patients attending the dermatology clinic at NewLab Clinical Research Inc. between 1989 and 2012. It is maintained by NewLab Clinical Research Inc. Data on psoriasis diagnosis and severity was obtained from this dataset.

The NLCHI Live Birth System for 1992-2012, which comprises clinical and demographic data on all live births in Newfoundland and Labrador, was used to identify birth records for women with and without psoriasis. A unique identifier was used to link the NewLab Psoriasis Clinical Dataset to the Live Birth System in order to identify births to women with psoriasis. Pregnancy outcomes data were also derived from this dataset.

The NLCHI Clinical Database Management System (CDMS) and the Newfoundland and Labrador Medical Care Plan (MCP) Fee for Service (FFS) Physician Claims Databases consist of hospital discharge data and data on physician services provided on a fee-for-service basis, respectively, for Newfoundland and Labrador. These datasets were used to check medical records of controls, to ensure that they had no documented psoriasis diagnosis. The control sample was drawn from birth records of women with no medical record in the NewLab Psoriasis Clinical Dataset and no record of psoriasis diagnosis or treatment in the CDMS or FFS.

Population estimate data for Newfoundland and Labrador for 2001-2013 were matched to the study dataset by Standard Geographical Classification (SGC) code, and used to create a variable for urban-rural residence.

\section{Study population}

Cases were women ages 18 to 49 who had been diagnosed with psoriasis between 1989 to 2012, as documented in the NewLab Psoriasis Clinical Database, with one or more documented live births in Newfoundland and Labrador between 1992 and 2012. Only patients diagnosed with psoriasis at least six months prior to giving birth were eligible for inclusion in the study. Patients with an unstable medical or surgical condition or other medical condition that might affect the conduct of the study, as well as patients with a history of drug or alcohol abuse, were excluded from the study.

Controls were selected from a population of women ages 18 to 49 without a diagnosis of psoriasis (as determined by the absence of a patient record in the NewLab Psoriasis Clinical Database or a diagnosis of psoriasis documented in the CDMS or FFS databases), who had given birth in Newfoundland and Labrador between 1992 and 2012. From the target population of controls, a sample of birth records matched to the birth records of psoriasis patients on maternal age, parity, and year of birth was selected. From that sample, a subsample was randomly selected to achieve a final study sample with a 1:4 ratio of births to psoriasis patients versus controls.

\section{Measures}

Exposures of interest were diagnosis of psoriasis and disease severity, as documented in the NewLab Psoriasis Clinical Database. Psoriasis severity was categorized as mild or moderate-to-severe, with the latter defined as psoriasis affecting at least $5 \%$ of the surface of a patient's body and/or psoriasis of the face, palms, or genital region [20].

Outcome measures were obtained from the NLCHI Live Birth Dataset and included delivery by Caesarean section, SGA, LBW (birth weight less than $2500 \mathrm{~g}$ ), PTB (prior to 37 weeks completed gestation), and diagnosis of any congenital anomaly at birth. A composite measure of overall negative outcome, comprising Caesarean delivery, SGA, LBW, and/or PTB was also considered. Due to the high proportion $(23.0 \%)$ of birth records missing data on congenital anomaly, this variable was not included in the composite outcome.

Adjustment variables for logistic regression analyses were maternal age, parity, child's sex, marital status (married versus unmarried), and urban-rural residence (defined as a population of $>1000$ versus 1000 or less).

\section{Data analysis}

Statistical analyses were performed using SAS version 9.3. Means for continuous variables were calculated and compared across psoriasis case status groups using Student's t-test. Frequencies were calculated for discrete variables and compared across case status and severity groups using Chi-square (as cell frequencies permitted) or Fisher's exact test. For the unadjusted analysis of pregnancy outcomes by disease severity, separate comparisons were made for outcomes in women with mild and moderate-to-severe psoriasis, using women without psoriasis as a reference group.

Multivariate logistic regression was performed to analyze the predictive effect of psoriasis case status and severity on pregnancy outcomes. A generalized estimating equation model, which allows the correlation of outcomes for a given individual to be estimated and accounted for in the calculation of regression coefficients and standard errors, [21] was used to take into account clustering of outcomes among births to the same mother. Adjustment was made for maternal age, parity, child's sex, marital status, and urban-rural residence. Maternal education was not included in the logistic regression models, as data on this variable were absent from the majority of birth records.

\section{Ethical and privacy considerations}

Ethics approval was obtained from the Human Research Ethics Authority of Newfoundland and Labrador and the NLCHI Secondary Uses Committee. All personal identifiers were removed from the linked dataset prior to analysis, and datasets were stored on a secure server at NLCHI.

\section{Results}

Of 1653 records of patients in the NewLab Psoriasis Clinical Database who were eligible for inclusion in the study, 385 could be linked to the NLCHI Live Birth Dataset for 1992-2012, giving a linkage rate of $23.3 \%$. This is a reasonable linkage rate, as not all eligible psoriasis patients would have given birth during the study time period.

The 385 psoriasis patients represented 615 births in the Live Birth Dataset. Following exclusion of one non-singleton birth, 2444 births to 2406 women without psoriasis were added to the linked dataset, for an 
approximate 1:4 ratio of births to psoriasis patients versus controls. The total sample therefore comprised 3059 live births.

Objective 1: To describe the demographic (marital status, urban/ rural residence, education) and reproductive (parity and age at first birth) characteristics of women with and without psoriasis.

Mean parity and age at first birth for psoriasis patients and controls are shown in Table 2. Psoriasis patients in this sample had a significantly higher parity, though this may be due to the process of record selection, as the study sample contained a higher percentage of multiple births to the same mother for psoriasis patients compared to controls.

Table 3 shows patients' marital status, urban-rural residence, and education level. No significant differences between psoriasis patients and controls were observed, although it should be noted that education data were missing for $59 \%$ of birth records.

Objective 2: To examine overall rates of adverse pregnancy outcomes among women with and without psoriasis.

Tables 4-9 show the unadjusted rates of Caesarean delivery, SGA, LBW, PTB, congenital anomaly, and any negative outcome (Caesarean, SGA, LBW, and/or PTB) for psoriasis patients and controls. In the unadjusted analysis, births to patients with psoriasis were shown to be

Table 1. Number of psoriasis patient records that linked to live birth records.

\begin{tabular}{|c|c|c|}
\hline $\begin{array}{c}\text { \# Eligible psoriasis } \\
\text { patients }\end{array}$ & $\begin{array}{c}\text { \# Records linked to live } \\
\text { births }\end{array}$ & \% Linkage \\
\hline 1653 & 385 & 23.3 \\
\hline
\end{tabular}

Table 2. Mean parity and age at first birth in psoriasis cases and controls.

\begin{tabular}{|c|c|c|c|c|c|}
\hline & \multicolumn{2}{|c|}{ Psoriasis $(n=385)$} & \multicolumn{2}{|c|}{ No psoriasis $(\mathrm{n}=\mathbf{2 4 0 6}$ ) } & \multirow{2}{*}{ p-value } \\
\hline & Mean & SD & Mean & SD & \\
\hline Parity* & 1.90 & 0.8 & 1.66 & 0.7 & $<0.0001 * *$ \\
\hline Age at first birth (years) & 27.96 & 5.2 & 28.71 & 5.0 & 0.01 \\
\hline
\end{tabular}

${ }^{a}$ p-value calculated using Student's t-test

*At most recent birth

$* * \mathrm{p}<0.05$

Table 3. Marital status, urban-rural residence, and education level of psoriasis patients and controls.

\begin{tabular}{|c|c|c|c|c|c|}
\hline & \multicolumn{2}{|c|}{ Psoriasis $(n=385)$} & \multicolumn{2}{|c|}{ No psoriasis $(n=2406)$} & \multirow[t]{2}{*}{ p-value ${ }^{a}$} \\
\hline & $\mathbf{n}$ & $\%$ & $\mathbf{n}$ & $\%$ & \\
\hline Currently married* & 253 & 65.7 & 1527 & 63.5 & 0.41 \\
\hline Urban residence* & 54 & 14.6 & 420 & 17.9 & 0.12 \\
\hline \multicolumn{6}{|l|}{ Education* } \\
\hline Less than high school & 12 & 7.0 & 82 & 8.3 & 0.78 \\
\hline High school graduate & 27 & 15.8 & 177 & 18.0 & \\
\hline Some college/university & 20 & 11.7 & 101 & 10.3 & \\
\hline College/university graduate & 112 & 65.5 & 623 & 63.4 & \\
\hline
\end{tabular}

${ }^{a} \mathrm{p}$-value calculated using Chi-square test for independence

*At most recent birth

Table 4. Unadjusted rates of Caesarean and vaginal delivery, psoriasis patients and controls

\begin{tabular}{|l|c|c|c|c|c|}
\hline \multirow{2}{*}{} & \multicolumn{2}{|c|}{ Caesarean delivery } & \multicolumn{2}{|c|}{ Vaginal delivery } & \multirow{2}{*}{ p-value $^{\mathbf{a}}$} \\
\cline { 1 - 5 } & $\mathbf{n}$ & $\mathbf{\%}$ & $\mathbf{n}$ & $\mathbf{\%}$ & \\
\hline Psoriasis $(\mathrm{N}=615)$ & 200 & 32.5 & 415 & 67.5 & \multirow{2}{*}{$0.03 * *$} \\
\hline No psoriasis $(\mathrm{n}=2444)$ & 688 & 28.1 & 1756 & 71.9 & \\
\hline
\end{tabular}

${ }^{a} \mathrm{p}$-value calculated using Chi-square test for independence $* * \mathrm{p}<0.05$
Table 5. Unadjusted rates of small for gestational age, psoriasis patients and controls*.

\begin{tabular}{|l|c|c|c|c|c|}
\hline \multirow{2}{*}{} & \multicolumn{2}{|c|}{ SGA } & \multicolumn{2}{c|}{ Not SGA } & \multirow{2}{*}{ p-value } \\
\cline { 2 - 5 } & n & \% & n & \% & \\
\hline Psoriasis $(\mathrm{n}=594)$ & 61 & 10.3 & 533 & 89.7 & 0.23 \\
\hline No psoriasis $(\mathrm{n}=2374)$ & 206 & 8.7 & 2168 & 91.3 & \\
\hline
\end{tabular}

${ }^{a}$ p-value calculated using Chi-square test for independence *Size for gestational age data were missing for 91 records

Table 6. Unadjusted rates of low birth weight, psoriasis patients and controls*.

\begin{tabular}{|l|c|c|c|c|c|}
\hline \multirow{2}{*}{} & \multicolumn{2}{|c|}{ LBW } & \multicolumn{2}{c|}{ Not LBW } & \multirow{2}{*}{ p-value $^{\mathbf{a}}$} \\
\cline { 2 - 5 } & $\mathbf{n}$ & $\mathbf{\%}$ & $\mathbf{n}$ & $\mathbf{\%}$ & \\
\hline Psoriasis (n=615) & 43 & 7 & 572 & 93 & 0.46 \\
\hline No psoriasis (n=2442) & 151 & 6.2 & 2291 & 93.8 & \\
\hline
\end{tabular}

${ }^{\mathrm{a}} \mathrm{p}$-value calculated using Chi-square test for independence

*Birth weight data were missing for two birth records.

Table 7. Unadjusted rates of preterm birth, psoriasis patients and controls*

\begin{tabular}{|l|c|c|c|c|c|}
\hline \multirow{2}{*}{} & \multicolumn{2}{|c|}{ PTB } & \multicolumn{2}{c|}{ Not PTB } & \multirow{2}{*}{ p-value $^{\mathbf{a}}$} \\
\cline { 2 - 5 } & $\mathbf{n}$ & $\mathbf{\%}$ & $\mathbf{n}$ & $\mathbf{\%}$ & \\
\hline Psoriasis (n=612) & 51 & 8.3 & 561 & 91.7 & 0.35 \\
\hline No psoriasis (n=2436) & 176 & 7.2 & 2260 & 92.8 & \\
\hline
\end{tabular}

${ }^{a}$ p-value calculated using Chi-square test for independence

*Gestational age data were missing for 11 birth records.

Table 8. Unadjusted rates of congenital anomaly, psoriasis patients and controls*

\begin{tabular}{|l|c|c|c|c|c|}
\hline \multirow{2}{*}{} & \multicolumn{2}{|c|}{ Congenital anomaly } & No congenital anomaly & \multirow{2}{*}{ p-value } \\
\cline { 2 - 5 } & $\mathbf{n}$ & $\mathbf{\%}$ & $\mathbf{n}$ & $\mathbf{\%}$ & \\
\hline Psoriasis $(\mathrm{n}=471)$ & 6 & 1.3 & 465 & 98.7 & 0.32 \\
\hline No psoriasis $(\mathrm{n}=1884)$ & 37 & 2 & 1847 & 98 & \\
\hline
\end{tabular}

${ }^{\mathrm{a}} \mathrm{p}$-value calculated using Chi-square test for independence

*Data on congenital anomaly were missing for 704 birth records $(23.0 \%)$.

Table 9: Unadjusted rates of overall negative pregnancy outcome, psoriasis patients and controls.

\begin{tabular}{|l|c|c|c|c|c|}
\hline \multirow{2}{*}{} & \multicolumn{2}{|c|}{ Negative outcome } & \multicolumn{2}{|c|}{ No negative outcome } & \multirow{2}{*}{ p-value -val $^{\mathbf{2}}$} \\
\cline { 2 - 5 } & $\mathbf{n}$ & $\mathbf{\%}$ & $\mathbf{n}$ & $\mathbf{\%}$ & \\
\hline Psoriasis $(\mathrm{n}=594)$ & 254 & 42.7 & 340 & 57.2 & \multirow{2}{*}{$0.01 * *$} \\
\hline No psoriasis $(\mathrm{n}=2374)$ & 880 & 37.1 & 1494 & 62.9 & \\
\hline
\end{tabular}

${ }^{a}$ p-value calculated using Chi-square test for independence

$* * \mathrm{p}<0.05$

significantly more likely to result in Caesarean delivery or any negative outcome (Tables 4 and $9, p=0.03$ and 0.01 , respectively), compared to births to women without psoriasis. Other individual outcome measures did not demonstrate significant differences between psoriasis patients and controls.

Objective 3: To examine overall rates of adverse pregnancy outcomes by psoriasis severity (mild or moderate-to-severe psoriasis vs. controls).

Tables 10-15 show the unadjusted rates of the above outcomes, with outcomes for psoriasis patients stratified by disease severity (mild or moderate-to-severe) and compared to those for women without psoriasis. Moderate-to-severe psoriasis was found to be associated with significantly higher rates of SGA or any negative outcome (Tables 11 
Table 10: Unadjusted rates of Caesarean and vaginal delivery, by psoriasis severity.

\begin{tabular}{|l|c|c|c|c|c|}
\hline \multirow{2}{*}{} & \multicolumn{2}{|c|}{ Caesarean delivery } & \multicolumn{2}{|c|}{ Vaginal delivery } & \multirow{2}{*}{ p-value -val $^{\mathbf{2}}$} \\
\cline { 2 - 6 } & $\mathbf{n}$ & $\mathbf{\%}$ & $\mathbf{n}$ & $\mathbf{\%}$ & \\
\hline No psoriasis (n=2444) & 688 & 28.2 & 1756 & 71.8 & ref \\
\hline Mild (n=243) & 75 & 30.9 & 168 & 69.1 & 0.37 \\
\hline Moderate/severe (n=260) & 86 & 33.1 & 174 & 66.9 & 0.09 \\
\hline
\end{tabular}

${ }^{\text {a }} \mathrm{p}$-value calculated using Chi-square test for independence

Table 11. Unadjusted rates of small for gestational age, by psoriasis severity*.

\begin{tabular}{|l|c|c|c|c|c|}
\hline \multirow{2}{*}{} & \multicolumn{2}{|c|}{ SGA } & \multicolumn{2}{c|}{ Not SGA } & \multirow{2}{*}{ p-value a $^{\mathbf{2}}$} \\
\cline { 2 - 6 } & $\mathbf{n}$ & $\mathbf{\%}$ & $\mathbf{n}$ & $\mathbf{\%}$ & \\
\hline No psoriasis (n=2374) & 206 & 8.7 & 2168 & 91.3 & ref \\
\hline Mild (=232) & 20 & 8.6 & 212 & 91.4 & 0.98 \\
\hline Moderate/severe (n=253) & 32 & 12.6 & 221 & 87.4 & $0.04 * *$ \\
\hline
\end{tabular}

${ }^{\text {ap}}$-value calculated using Chi-square test for independence $*$ Size for gestational age data were missing for 91 birth records $* * \mathrm{p}<0.05$

Table 12. Unadjusted rates of low birth weight, by psoriasis severity*.

\begin{tabular}{|l|c|c|c|c|c|}
\hline \multirow{2}{*}{} & \multicolumn{2}{|c|}{ LBW } & \multicolumn{2}{c|}{ Not LBW } & \multirow{2}{*}{ p-value ${ }^{\mathbf{a}}$} \\
\cline { 2 - 6 } & $\mathbf{n}$ & $\mathbf{\%}$ & $\mathbf{n}$ & $\mathbf{\%}$ & \\
\hline No psoriasis (n=2442) & 151 & 6.2 & 2291 & 93.8 & ref \\
\hline Mild (n=243) & 17 & 7 & 226 & 93 & 0.62 \\
\hline Moderate/severe (n=260) & 19 & 7.3 & 241 & 92.7 & 0.48 \\
\hline
\end{tabular}

${ }^{a} \mathrm{p}$-value calculated using Chi-square test for independence

*Birth weight data were missing for two birth records

Table 13: Unadjusted rates of preterm birth, by psoriasis severity*.

\begin{tabular}{|l|c|c|c|c|c|}
\hline \multirow{2}{*}{} & \multicolumn{2}{|c|}{ PTB } & \multicolumn{2}{c|}{ Not PTB } & \multirow{2}{*}{-value $^{\text {a }}$} \\
\cline { 1 - 5 } & $\mathbf{n}$ & $\mathbf{\%}$ & $\mathbf{n}$ & $\mathbf{\%}$ & \\
\hline No psoriasis (n=2436) & 176 & 7.2 & 2260 & 92.8 & ref \\
\hline Mild (n=243) & 24 & 9.9 & 219 & 90.1 & 0.13 \\
\hline Moderate/severe (n=257) & 17 & 6.6 & 240 & 93.4 & 0.72 \\
\hline
\end{tabular}

${ }^{\text {a }}$-value calculated using Chi-square test for independence

*Gestational age data were missing for 11 birth records

Table 14. Unadjusted rates of congenital anomaly, by psoriasis severity*.

\begin{tabular}{|l|c|c|c|c|c|}
\hline \multirow{2}{*}{} & \multicolumn{2}{|c|}{ Congenital anomaly } & \multicolumn{2}{c|}{ No congenital anomaly } & \multirow{2}{*}{ p-value ${ }^{\mathbf{b}}$} \\
\cline { 2 - 5 } & $\mathbf{n}$ & $\mathbf{\%}$ & $\mathbf{n}$ & $\mathbf{\%}$ & \\
\hline No psoriasis $(\mathrm{n}=1884)$ & 37 & 2 & 1847 & 98 & ref \\
\hline Mild (n=190) & 3 & 1.6 & 187 & 98.4 & 1 \\
\hline Moderate/severe $(\mathrm{n}=202)$ & 2 & 1 & 200 & 99 & 0.58 \\
\hline
\end{tabular}

${ }^{b} \mathrm{p}$-value calculated using Fisher's exact test

*Data on congenital anomaly were missing for 704 birth records (23.0\%).

and $15, \mathrm{p}=0.04$ and 0.004 , respectively), although disease severity data were not available for $112(18.2 \%)$ of births to women with psoriasis.

Objective 4: To analyze psoriasis as a predictor of pregnancy outcomes, controlling for potential confounding factors.

Tables 16-21 show the results of a logistic regression analysis of psoriasis as a predictor of pregnancy outcomes, with generalized estimating equations used to correct for clustering of outcomes of pregnancies to the same mother. After controlling for the effects of parity, maternal age, child's sex, mother's marital status and urbanrural residence, psoriasis did not emerge as a significant predictor of any individual pregnancy outcome or of the composite negative outcome, although the association of psoriasis with the latter outcome approached statistical significance (Table 21, $\mathrm{p}=0.055$ ).

Table 15. Unadjusted rates of overall negative pregnancy outcome, by psoriasis severity.

\begin{tabular}{|l|c|c|c|c|c|}
\hline \multirow{2}{*}{} & \multicolumn{2}{|c|}{ Negative outcome } & \multicolumn{2}{|c|}{ No negative outcome } & \multirow{2}{*}{ p-value a $^{\mathbf{2}}$} \\
\cline { 2 - 5 } & $\mathbf{n}$ & $\mathbf{\%}$ & $\mathbf{n}$ & $\mathbf{\%}$ & \\
\hline No psoriasis $(\mathrm{n}=2374)$ & 880 & 37.1 & 1494 & 62.9 & ref \\
\hline Mild (n=232) & 88 & 37.9 & 144 & 62.1 & 0.8 \\
\hline Moderate/severe $(\mathrm{n}=253)$ & 117 & 46.2 & 136 & 53.8 & $0.004 * *$ \\
\hline
\end{tabular}

ap-value calculated using Chi-square test for independence

$* * \mathrm{p}<0.05$

Table 16. Adjusted model of psoriasis as a predictor of Caesarean delivery.

\begin{tabular}{|l|l|l|l|}
\hline b & OR & $\mathbf{9 5 \%}$ CI & p-value \\
\hline Psoriasis case & 1.04 & $0.99,1.10$ & 0.12 \\
\hline Number of live births & 0.96 & $0.94,0.98$ & $<0.0001$ \\
\hline Maternal age & 1.02 & $1.01,1.02$ & $<0.0001$ \\
\hline Child' sex (female $v s$. male) & 0.99 & $0.96,1.03$ & 0.63 \\
\hline Marital status (married $v s$. unmarried) & 1.01 & $0.97,1.05$ & 0.67 \\
\hline Residence (rural $v s$. urban) & 0.98 & $0.93,1.02$ & 0.33 \\
\hline
\end{tabular}

Table 17. Adjusted model of psoriasis as a predictor of small-for-gestational age.

\begin{tabular}{|l|l|l|l|}
\hline & OR & $\mathbf{9 5 \%}$ CI & p-value \\
\hline Psoriasis case & 1.01 & $0.98,1.04$ & 0.42 \\
\hline Number of live births & 0.98 & $0.97,1.00$ & 0.03 \\
\hline Maternal age & 1 & $1.00,1.00$ & 0.91 \\
\hline Child' sex (female $v s$. male) & 0.98 & $0.96,1.00$ & 0.08 \\
\hline Marital status (married $v s$. unmarried) & 0.96 & $0.93,0.98$ & 0.0004 \\
\hline Residence (rural $v s$. urban) & 1 & $0.97,1.03$ & 0.89 \\
\hline
\end{tabular}

Table 18. Adjusted model of psoriasis as a predictor of low birth weight.

\begin{tabular}{|l|l|l|l|}
\hline & OR & $\mathbf{9 5 \%}$ CI & p-value \\
\hline Psoriasis case & 1.01 & $0.98,1.03$ & 0.57 \\
\hline Number of live births & 1 & $0.99,1.01$ & 0.98 \\
\hline Maternal age & 1 & $1.00,1.00$ & 0.1 \\
\hline Child' sex (female $v$ s. male) & 0.98 & $0.97,1.00$ & 0.09 \\
\hline Marital status (married $v s$. unmarried) & 0.97 & $0.95,0.00$ & 0.002 \\
\hline Residence (rural $v s$. urban) & 1.02 & $1.00,1.05$ & 0.04 \\
\hline
\end{tabular}

Table 19. Adjusted model of psoriasis as a predictor of preterm birth.

\begin{tabular}{|l|l|l|l|}
\hline & OR & $\mathbf{9 5 \%}$ CI & p-value \\
\hline Psoriasis case & 1.01 & $0.98,1.04$ & 0.41 \\
\hline Number of live births & 0.99 & $0.98,1.01$ & 0.4 \\
\hline Maternal age & 1 & $1.00,1.00$ & 0.03 \\
\hline Child' sex (female $v s$. male) & 0.97 & $0.95,0.99$ & 0.002 \\
\hline Marital status (married $v s$. unmarried) & 0.98 & $0.96,1.00$ & 0.03 \\
\hline Residence (rural $v s$. urban) & 1.03 & $1.01,1.05$ & 0.01 \\
\hline
\end{tabular}

Table 20. Adjusted model of psoriasis as a predictor of congenital anomaly.

\begin{tabular}{|l|l|l|l|}
\hline & OR & $\mathbf{9 5 \%}$ CI & p-value \\
\hline Psoriasis case & 1.01 & $0.99,1.03$ & 0.15 \\
\hline Number of live births & 0.99 & $0.98,1.00$ & 0.19 \\
\hline Maternal age & 1 & $1.00,1.01$ & 0.004 \\
\hline Child' sex (female $v s$. male) & 1.01 & $1.00,1.03$ & 0.08 \\
\hline Marital status (married $v s$. unmarried) & 0.97 & $0.96,0.99$ & 0.007 \\
\hline Residence (rural $v s$. urban) & 1 & $0.98,1.01$ & 0.68 \\
\hline
\end{tabular}


Table 21. Adjusted model of psoriasis as a predictor of overall negative outcome.

\begin{tabular}{|l|l|l|l|}
\hline & OR & $\mathbf{9 5 \%}$ CI & p-value \\
\hline Psoriasis case & 1.05 & $0.999,1.11$ & 0.055 \\
\hline Number of live births & 0.94 & $0.92,0.96$ & $<0.0001$ \\
\hline Maternal age & 1.02 & $1.01,1.02$ & $<0.0001$ \\
\hline Child' sex (female $v s$. male) & 0.96 & $0.92,0.99$ & 0.01 \\
\hline Marital status (married $v s$. unmarried) & 0.95 & $0.92,0.99$ & 0.03 \\
\hline Residence (rural $v s$. urban) & 0.99 & $0.94,1.04$ & 0.69 \\
\hline
\end{tabular}

Table 22. Adjusted model of psoriasis severity as a predictor of Caesarean delivery.

\begin{tabular}{|l|l|l|l|}
\hline & OR & $\mathbf{9 5 \%}$ CI & p-value \\
\hline Psoriasis case & 1.03 & $0.99,1.06$ & 0.14 \\
\hline Number of live births & 0.96 & $0.94,0.98$ & $<0.0001$ \\
\hline Maternal age & 1.02 & $1.01,1.02$ & $<0.0001$ \\
\hline Child' sex (female $v s$. male) & 0.99 & $0.96,1.02$ & 0.58 \\
\hline Marital status (married $v$ s. unmarried) & 1.00 & $0.97,1.04$ & 0.89 \\
\hline Residence (rural $v s$. urban) & 0.97 & $0.93,1.02$ & 0.26 \\
\hline
\end{tabular}

Table 23. Adjusted model of psoriasis severity as a predictor of small-for-gestational age.

\begin{tabular}{|l|l|l|l|}
\hline & OR & $\mathbf{9 5 \%}$ CI & p-value \\
\hline Psoriasis case & 1.02 & $0.99,1.04$ & 0.17 \\
\hline Number of live births & 0.98 & $0.97,1.00$ & 0.01 \\
\hline Maternal age & 1.00 & $1.00,1.00$ & 0.98 \\
\hline Child' sex (female $v s$. male) & 0.98 & $0.96,1.00$ & 0.08 \\
\hline Marital status (married $v s$. unmarried) & 0.96 & $0.94,0.98$ & 0.001 \\
\hline Residence (rural $v s$. urban) & 1.00 & $0.97,1.03$ & 0.85 \\
\hline
\end{tabular}

Table 24. Adjusted model of psoriasis severity as a predictor of low birth weight.

\begin{tabular}{|l|l|l|l|}
\hline & OR & $\mathbf{9 5 \%}$ CI & p-value \\
\hline Psoriasis case & 1.01 & $0.99,1.02$ & 0.51 \\
\hline Number of live births & 1.00 & $0.99,1.01$ & 0.95 \\
\hline Maternal age & 1.00 & $1.00,1.00$ & 0.09 \\
\hline Child' sex (female $v s$. male) & 0.98 & $0.97,1.00$ & 0.07 \\
\hline Marital status (married $v s$. unmarried) & 0.97 & $0.95,0.99$ & 0.003 \\
\hline Residence (rural $v s$. urban) & 1.02 & $1.00,1.05$ & 0.04 \\
\hline
\end{tabular}

Table 25. Adjusted model of psoriasis severity as a predictor of preterm birth.

\begin{tabular}{|l|l|l|l|}
\hline & OR & $\mathbf{9 5 \%}$ CI & p-value \\
\hline Psoriasis case & 1.00 & $0.98,1.02$ & 0.8 \\
\hline Number of live births & 1.00 & $0.98,1.01$ & 0.57 \\
\hline Maternal age & 1.00 & $1.00,1.00$ & 0.05 \\
\hline Child' sex (female $v$ s. male) & 0.97 & $0.95,0.99$ & 0.0005 \\
\hline Marital status (married $v$ s. unmarried) & 0.98 & $0.96,1.00$ & 0.06 \\
\hline Residence (rural $v s$. urban) & 1.03 & $1.01,1.05$ & 0.01 \\
\hline
\end{tabular}

Table 26. Adjusted model of psoriasis severity as a predictor of congenital anomaly.

\begin{tabular}{|l|l|l|l|}
\hline & OR & $\mathbf{9 5 \%}$ CI & p-value \\
\hline Psoriasis case & 1.01 & $1.00,1.02$ & 0.19 \\
\hline Number of live births & 0.99 & $0.98,1.00$ & 0.21 \\
\hline Maternal age & 1.00 & $1.00,1.01$ & 0.004 \\
\hline Child' sex (female $v s$. male) & 1.01 & $1.00,1.03$ & 0.07 \\
\hline Marital status (married $v$ s. unmarried) & 0.97 & $0.95,0.99$ & 0.004 \\
\hline Residence (rural $v s$. urban) & 1.00 & $0.98,1.02$ & 0.88 \\
\hline
\end{tabular}

Table 27. Adjusted model of psoriasis severity as a predictor of overall negative outcome.

\begin{tabular}{|l|l|l|l|}
\hline & OR & $\mathbf{9 5 \%}$ CI & p-value \\
\hline Psoriasis case & 1.04 & $1.005,1.08$ & $0.03 * *$ \\
\hline Number of live births & 0.94 & $0.91,0.96$ & $<0.0001$ \\
\hline Maternal age & 1.01 & $1.01,1.02$ & $<0.0001$ \\
\hline Child' sex (female $v s$. male) & 0.96 & $0.92,0.99$ & 0.01 \\
\hline Marital status (married $v s$. unmarried) & 0.95 & $0.92,0.99$ & 0.02 \\
\hline Residence (rural $v$ s. urban) & 0.98 & $0.94,1.03$ & 0.44 \\
\hline
\end{tabular}

${ }^{* *} \mathrm{p}<0.05$

Objective 5: To analyze psoriasis severity as a predictor of pregnancy outcomes, controlling for potential confounding factors.

Similarly, as shown in tables 22-27, logistic regression analysis did not show psoriasis severity to be a significant predictor of any individual pregnancy outcome. However, psoriasis severity was significantly associated with the composite negative outcome (Table 27, $\mathrm{p}=0.03$ ).

\section{Discussion}

The current analysis contributes to the existing literature on pregnancy outcomes among women with psoriasis. Prior studies on this topic are few, and several have been limited by small study samples [6]. Moreover, the majority of early studies comprised only patients with mild psoriasis, and could not assess the effect of moderate-tosevere disease on pregnancy outcomes [5].

We observed an association of psoriasis severity with an overall negative pregnancy outcome but not with individual outcomes. Similar findings were reported by Lima et al. [6] who reported an association of psoriasis with a composite outcome comprising PTB and LBW, but not with either individual outcome. The authors hypothesized that their sample was adequate to detect an association with the composite outcome but insufficient to detect associations with individual outcomes. While the current study involved a larger sample than that of Lima et al., it is possible that the relative infrequency of some individual outcomes in our study sample limited our ability to detect associations with these outcomes, particularly when the analysis was stratified by disease severity.

To our knowledge, only two prior studies have previously examined the effect of moderate-to-severe psoriasis on pregnancy. In a matched cohort study of pregnancy outcomes in women with moderate-to-severe psoriasis and women without psoriasis, CohenBarak et al. [5] reported several adverse pregnancy outcomes associated with moderate-to-severe psoriasis, including spontaneous abortion, hypertensive diseases of pregnancy, premature rupture of membranes, macrosomia, and large for gestational age. However, their analysis failed to account for clustering of outcomes across pregnancies to the same mother. Further, all study participants were recruited from a single university medical centre [5], and both cases and controls may have been at higher-than-average risk of adverse pregnancy outcomes, thereby potentially biasing the findings for certain outcomes toward the null [18].

Yang et al. [18] conducted a population-based analysis of the effect of psoriasis case status and severity on pregnancy outcomes, utilizing linked national health insurance and birth certificate registry data from Taiwan. The authors found an increased risk of LBW $(\mathrm{OR}=1.40)$ among women with moderate-to-severe psoriasis, while mild psoriasis did not demonstrate an association with any negative pregnancy outcome. Strengths of the work of Yang et al. include its sample size $(\mathrm{N}=1463$ 
births to women with psoriasis) and population-based design, but the study is limited in the potential for exposure misclassification resulting from the use of diagnostic codes to identify psoriasis patients, and in the potential for residual confounding due to unmeasured maternal factors [18].

The current analysis involved use of a patient clinical database to identify disease status and severity; therefore, exposure misclassification is less of a concern for our analysis. However, like Yang et al., we lacked data on maternal comorbidities and lifestyle factors that may contribute to adverse pregnancy outcomes in women with psoriasis. Of particular interest is the well-documented association between psoriasis and obesity [4,22-24]. It has been hypothesized that production of inflammatory cytokines by adipose tissue may contribute to the development of psoriasis [25], and a prospective cohort analysis found that the risk of psoriasis incidence increased with increasing body mass index [23], Prepregnancy obesity has been shown to be associated with risk of congenital anomalies [26], preterm birth [27], and Caesarean delivery $[28,29]$; thus, obesity is a potential confounder of any analysis of psoriasis and pregnancy outcomes.

Other comorbidities and lifestyle factors that have been demonstrated to be associated with psoriasis include hypertension $[22,30]$, diabetes $[22,30,31]$, depression [32] higher rates of smoking during pregnancy [4], and lower rates of prenatal vitamin use, 4 all of which may contribute to adverse pregnancy outcomes. Maternal depression has been linked to increased rates of PTB [33,34], SGA [34], and LBW [34]. Smoking during pregnancy is associated with intrauterine growth restriction [35] and increased rates of LBW [36] and PTB [37]. Use of prenatal vitamins containing folic acid has been shown to protect against neural tube defects [38] and may also confer a protective effect against pre-eclampsia and SGA [39]. The absence of data on comorbidities and lifestyle factors is therefore a significant limitation of our analysis, as residual confounding due to these factors may well contribute to the association between psoriasis severity and overall negative outcome that we observed.

A further limitation of the analysis is our inability to adjust for the effect of medical treatment for psoriasis on pregnancy outcomes. While data on the safety of many psoriasis treatments during pregnancy are lacking [7], we cannot exclude the possibility that the observed higher rates of adverse outcomes observed associated with psoriasis severity in this analysis may be due in part to the effects of treatment for psoriasis, rather than to the condition itself.

Finally, a high percentage of records were missing data on the disease severity among psoriasis patients and on the presence of congenital anomaly at birth. The degree of missing data on psoriasis severity may have limited our ability to detect associations between moderate-to-severe psoriasis and individual outcomes, while a high rate of missing data on the presence of congenital anomaly limited our ability to detect associations with this outcome or to include it in the composite outcome.

\section{Conclusions}

The current analysis adds to the existing literature on pregnancy outcomes among women with psoriasis and suggests that psoriasis severity may be predictive of overall negative pregnancy outcomes. However, our ability to draw conclusions about the effect of psoriasis on pregnancy outcomes is limited by the absence of data on comorbidities, lifestyle factors, or psoriasis treatment. Further research is warranted to control for the effects of psoriasis comorbidities and treatment on the association between psoriasis severity and adverse pregnancy outcomes.

\section{Funding}

This project received an unrestricted study grant from Janssen Pharmaceutica.

\section{References}

1. Gottlieb AB, Chao C, Dann F (2008) Psoriasis comorbidities. J Dermatolog Treat 19: 5-21. [Crossref]

2. Parisi R, Symmons DP, Griffiths CE, Ashcroft DM; Identification and Management of Psoriasis and Associated ComorbidiTy (IMPACT) project team (2013) Global epidemiology of psoriasis: a systematic review of incidence and prevalence. J Invest Dermatol 133: 377-385. [Crossref]

3. Levine D, Gottlieb A (2009) Evaluation and management of psoriasis: an internist's guide. Med Clin North Am 93: 1291-1303. [Crossref]

4. Bandoli G, Johnson DL, Jones KL, Lopez Jiminez J, Salas E, et al. (2010) Potentially modifiable risk factors for adverse pregnancy outcomes in women with psoriasis. $\mathrm{BrJ}$ Dermatol 163: 334-339. [Crossref]

5. Cohen-Barak E, Nachum Z, Rozenman D, Ziv M (2011) Pregnancy outcomes in women with moderate-to-severe psoriasis. J Eur Acad Dermatol Venereol 25: 10411047. [Crossref]

6. Lima XT, Janakiraman V, Hughes MD, Kimball AB (2012) The impact of psoriasis on pregnancy outcomes. J Invest Dermatol 132: 85-91. [Crossref]

7. Horn EJ, Chambers CD, Menter A, Kimball AB; International Psoriasis Council (2009) Pregnancy outcomes in psoriasis: why do we know so little? J Am Acad Dermatol 61: e5-8. [Crossref]

8. Schön MP, Boehncke WH (2005) Psoriasis. N Engl J Med 352: 1899-1912. [Crossref]

9. Arican O, Aral M, Sasmaz S, Ciragil P (2005) Serum levels of TNF-alpha, IFN-gamma, IL-6, IL-8, IL-12, IL-17, and IL-18 in patients with active psoriasis and correlation with disease severity. Mediators Inflamm 2005: 273-279. [Crossref]

10. Guven MA, Coskun A, Ertas IE, Aral M, Zencirci B, et al. (2009) Association of maternal serum CRP, IL-6, TNF-alpha, homocysteine, folic acid and vitamin B12 levels with the severity of preeclampsia and fetal birth weight. Hypertens Pregnancy 28: 190-200. [Crossref]

11. Fried M, Muga RO, Misore AO, Duffy PE (1998) Malaria elicits type 1 cytokines in the human placenta: IFN-gamma and TNF-alpha associated with pregnancy outcomes. J Immunol 160: 2523-2530. [Crossref]

12. Dominitz JA, Young JC, Boyko EJ (2002) Outcomes of infants born to mothers with inflammatory bowel disease: a population-based cohort study. Am J Gastroenterol 97: 641-648. [Crossref]

13. Mahadevan U, Sandborn WJ, Li DK, Hakimian S, Kane S, et al. (2007) Pregnancy outcomes in women with inflammatory bowel disease: a large community-based study from Northern California. Gastroenterol 133: 1106-1112. [Crossref]

14. Bush MC, Patel S, Lapinski RH, Stone JL (2004) Perinatal outcomes in inflammatory bowel disease. J Matern Fetal Neonatal Med 15: 237-241. [Crossref]

15. Reed SD, Vollan TA, Svec MA (2006) Pregnancy outcomes in women with rheumatoid arthritis in Washington State. Matern Child Health J 10: 361-366. [Crossref]

16. Rom AL, Wu CS, Olsen J, Kjaergaard H, Jawaheer D, et al. (2014) Fetal growth and preterm birth in children exposed to maternal or paternal rheumatoid arthritis: a nationwide cohort study. Arthritis Rheumatol 66: 3265-3273. [Crossref]

17. Bowden AP, Barrett JH, Fallow W, Silman AJ (2001) Women with inflammatory polyarthritis have babies of lower birth weight. J Rheumatol 28: 355-359. [Crossref]

18. Yang YW, Chen CS, Chen YH, Lin HC (2011) Psoriasis and pregnancy outcomes: a nationwide population-based study. J Am Acad Dermatol 64: 71-77. [Crossref]

19. Ben David G, Sheiner E, Hallak M, Levi A (2005) Pregnancy outcome of patients with psoriasis. Am J Obstet Gynecol 193: S106. [Crossref]

20. Gulliver WP, Macdonald D, Gladney N, Alaghehbandan R, Rahman P, et al. (2011) Long-term prognosis and comorbidities associated with psoriasis in the Newfoundland and Labrador founder population. J Cutan Med Surg 15: 37-47. [Crossref]

21. Burton P, Gurrin L, Sly P (1998) Extending the simple linear regression model to 
account for correlated responses: an introduction to generalized estimating equations and multi-level mixed modelling. Stat Med 17: 1261-1291. [Crossref]

22. Sommer DM, Jenisch S, Suchan M, Christophers E, Weichenthal M (2006) Increased prevalence of the metabolic syndrome in patients with moderate to severe psoriasis. Arch Dermatol Res 298: 321-328. [Crossref]

23. Setty AR, Curhan G, Choi HK (2007) Obesity, waist circumference, weight change, and the risk of psoriasis in women: Nurses' Health Study II. Arch Intern Med 167: 1670-1675. [Crossref]

24. Armstrong AW, Harskamp CT, Armstrong EJ (2012) The association between psoriasis and obesity: a systematic review and meta-analysis of observational studies. Nutrition Diabetes 2: e54. [Crossref]

25. Hamminga EA, van der Lely AJ, Neumann HA, Thio HB (2006) Chronic inflammation in psoriasis and obesity: implications for therapy. Med Hypotheses 67: 768-773. [Crossref]

26. Waller DK, Shaw GM, Rasmussen SA, Hobbs CA, Canfield MA, et al. (2007) Prepregnancy obesity as a risk factor for structural birth defects. Arch Pediatr Adolesc Med 161: 745-750. [Crossref]

27. McDonald SD, Han Z, Mulla S, Beyene J (2010) Overweight and obesity in mothers and risk of preterm birth and low birth weight infants: systematic review and metaanalysis. $B M J 341$ : c3248. [Crossref]

28. Sheiner E, Levy A, Menes TS, Silverberg D, Katz M, et al. (2004) Maternal obesity as an independent risk factor for caesarean delivery. Paediatr Perinat Epidemiol 18 196-201. [Crossref]

29. Khashan AS, Kenny LC (2009) The effects of maternal body mass index on pregnancy outcome. Eur J Epidemiol 24: 697-705. [Crossref]

30. Neimann AL, Shin DB, Wang X, Margolis DJ, Troxel AB, et al. (2006) Prevalence of cardiovascular risk factors in patients with psoriasis. J Am Acad Dermatol 55: 829835. [Crossref]

31. Azfar RS, Seminara NM, Shin DB, Troxel AB, Margolis DJ, et al. (2012) Increased risk of diabetes mellitus and likelihood of receiving diabetes mellitus treatment in patients with psoriasis. Arch Dermatol 148: 995-1000. [Crossref]

32. Dowlatshahi EA, Wakkee M, Arends LR, Nijsten T (2014) The prevalence and odds of depressive symptoms and clinical depression in psoriasis patients: a systematic review and meta-analysis. J Invest Dermatol 134: 1542-1551. [Crossref]

33. Wisner KL, Sit DK, Hanusa BH, Moses-Kolko EL, Bogen DL, et al. (2009) Major depression and antidepressant treatment: impact on pregnancy and neonatal outcomes. Am J Psychiatry 166: 557-566. [Crossref]

34. Steer RA, Scholl TO, Hediger ML, Fischer RL (1992) Self-reported depression and negative pregnancy outcomes. J Clin Epidemiol 45: 1093-1099. [Crossref]

35. Murphy JF, Drumm JE, Mulcahy R, Daly L (1980) The effect of maternal cigarette smoking on fetal birth weight and on growth of the fetal biparietal diameter. $\mathrm{Br} J$ Obstet Gynaecol 87: 462-466. [Crossref]

36. Kramer MS (1987) Determinants of low birth weight: methodological assessment and meta-analysis. Bull World Health Organ 65: 663-737. [Crossref]

37. Shah NR, Bracken MB (2000) A systematic review and meta-analysis of prospective studies on the association between maternal cigarette smoking and preterm delivery. Am J Obstet Gynecol 182: 465-472. [Crossref]

38. De-Regil LM, Fernández-Gaxiola AC, Dowswell T, Peña-Rosas JP (2010) Effects and safety of periconceptional folate supplementation for preventing birth defects. Cochrane Database Syst Rev: CD007950. [Crossref]

39. Kim MW, Ahn KH, Ryu KJ, Hong SC, Lee JS, et al. (2014) Preventive effects of folic acid supplementation on adverse maternal and fetal outcomes. PLoS One 9: e97273. [Crossref]

Copyright: (C2015 Gulliver W. This is an open-access article distributed under the terms of the Creative Commons Attribution License, which permits unrestricted use, distribution, and reproduction in any medium, provided the original author and source are credited. 